\title{
The Application of the Key Technology of the Internet of Things
}

\author{
Yixiang Jiang ${ }^{a}$, Zhihui Ye ${ }^{b}$, Wenjuan Wang \\ China Tobacco Zhejiang Industrial CO., LTD. Zhejiang. China \\ ajiangyx@zjtobacco.com, byezhihui@zjtobacco.com
}

\begin{abstract}
The Internet of things (IOT) is a new achievement in the development and application of computer information technology, which promotes the convenience and intellectualization of people's daily life. The Internet of things mainly realizes the intelligent communication between machines, that is to say, it does not require human involvement to complete various tasks. The technical core of the Internet of things is as follows: embedding the networked information sensor into the object, controlling the sensor through the computer information and then commands the object to realize some function. The emergence of the Internet of things symbolizes the development of computer technology to a new stage. It has a very broad prospects for development. Firstly, this paper explains the concept and characteristics of the Internet of things. Secondly, it focuses on some key technologies and applications of the Internet of things.
\end{abstract}

Keywords: Internet of things, perception layer technology, network layer technology, application.

\section{Basic Concepts and Features of the Internet of Things}

\subsection{Concepts}

The emergence and development of the Internet of things marked the evolution of computer information technology to a new stage. As a representative product of information technology, the Internet of things has greatly facilitated people's life and contributed to the realization of "intelligent life". The English name of the Internet of things is "Internet of things". In terms of the meaning of Chinese, the Internet of things refers to the network formed by connecting various objects. The concept of the Internet of things is mainly manifested in two aspects. First, the basic and core technology of the Internet of things is computer Internet technology, and computer Internet technology (Information Technology) is the basic skeleton and technical support of the Internet, that is to say, the Internet of things is a new network structure which is extended and developed on the Internet [1]. Second, the user interface of the Internet of things has been transformed. The Internet of things is the main construction of the Internet of things, which is based on the computer Internet technology to transmit information between objects and objects. At this stage. Various advanced communication technologies, such as intelligent induction technology and identification technology, are widely used in the Internet of things.

\subsection{Features}

The emergence of the Internet of things is a great gospel for the development of human society, which directly promotes the improvement of people's production and life style, and also effectively improves the quality and efficiency of production. The features of the Internet of things are concentrated in two aspects. First, the huge development benefits of the Internet of things. At this stage, it is generally believed that the Internet of things is a great driving force for the rapid development of the world. The huge development benefit of the Internet of things is now "emerging". On the one hand, the construction and application of the Internet of things have subverted the traditional mode of production and life to a great extent. The work that needs manual responsibility in the past can be completed through the Internet of things [2] This has saved a lot of production cost for the enterprise, and also raised the life of the enterprise. Productivity and economic benefits. On the other hand, the development and popularization of the Internet of things will not bring up a phenomenon of factory and enterprise staff in a large area. On the contrary, it can provide a large number of technical posts to relieve the pressure of the employment of the society. According to the 
needs of the Internet of things, the production of hundreds of millions of electronic labels and sensors will be put on the agenda, which undoubtedly creates a large number of technical posts to solve the problem of employment. Second, the "innovation 2 mode" of the Internet of things development. The Internet of things is the extension and expansion of the Internet. On the one hand, it has the basic attributes of network architecture, and on the other hand, it pays more attention to the use of business. "Innovation 2 model" is a significant feature of the Internet of things development, which is driven by information technology and theoretical knowledge to promote the innovation and development of business. With the popularization and application of Internet of things technology, it has gradually formed the development pattern of "user centered, people-oriented, service society".

\section{Key Technologies and Applications of the Internet of Things}

\subsection{Sensor Technology and Its Application}

Sensor is a signal conversion device, it can achieve the conversion between signals and instructions. Sensors are generally composed of two parts, which are sensitive elements and conversion elements. The basic principle of sensor work is signal conversion, and the sensitive elements in the sensor convert various forms of signal (such as pressure signal, light intensity signal, temperature signal, etc.) into electrical signals, and then facilitate the recognition and processing of the computer information system. According to the different functions of sensors, sensors are divided into various categories: chemical sensors, mechanical sensors, optical sensors and acoustic sensors. Sensor technology is widely used in the Internet of things. It is no exaggeration to say that sensor technology is the second important technical support for Internet of things. At present, the application of sensor technology in the Internet of things covers aerospace, military technology and agricultural production. Taking the most common automatic door in life as an example, it is the use of sensor technology (through the induction of infrared signals to control the door switch); a variety of forms of alarm (such as smoke alarm, humidity alarm and water level alarm, etc.) are applied to the sensor technology. The application of sensor technology in the Internet of things has promoted the leap forward development of the function of the Internet of things, which is an important window for the Internet of things to perceive the external environment and obtain information. The innovation of sensor technology is complementary to the development of the Internet of things. To promote the development of the Internet of things, we must focus on improving and innovating sensor technology.[3]

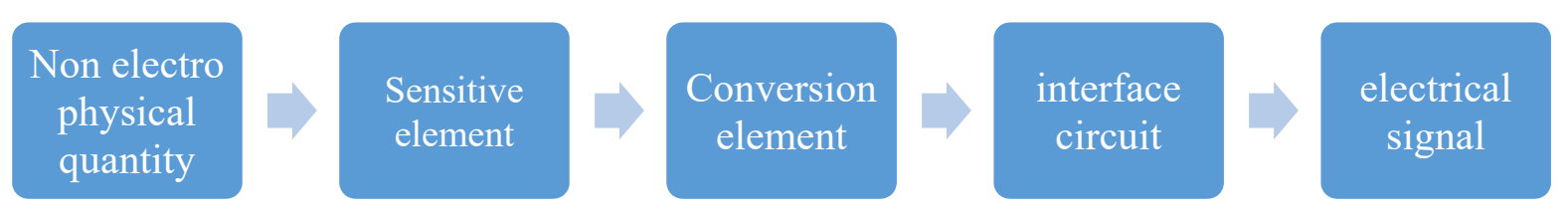

Fig.1 The Basic principles of sensor technology

\subsection{ZigBee Technology and Its Application}

ZigBee technology is essentially a short-range, low-power wireless communication technology, which has been widely used in the Internet of things. ZigBee technology has many advantages, such as large network capacity, short time delay, low work consumption and low cost. The following figure describes a series of advantages and performance of ZieBee technology [4].

Table 1.Introduction to the characteristics of ZigBee Technology

\begin{tabular}{cc}
\hline Characteristic & describe \\
\hline Large network capacity & Support more than 250 kinds of equipment \\
Time delay short & $15 \mathrm{~ms}-30 \mathrm{~ms}$ \\
Low consumption & Two months of battery 5 can be used for half a year \\
High security & Support data integrity test \\
Multiband & $868 \mathrm{MHz}, 915 \mathrm{MHz}, 2.4 \mathrm{GHz}$ \\
\hline
\end{tabular}


ZieBee technology has narrow communication scope and low data transmission rate. It has many applications in the small area of the Internet of things. At this stage, ZigBee technology has been more mature in family life automation, medical detection, agricultural detection, industrial control and other fields. Because of the low power and cost required by ZieBee technology, it has wider application space.

\subsection{RFID Technology and Its Application}

RFID technology, radio frequency identification technology, belongs to a type of automatic identification. RFID technology uses radio frequency method to carry out non-contact two-way data communication to identify target objects and obtain relevant data information. The implementation of RFID technology needs to be dependent on the RFID system, a complete RFID system consists of three parts of the structure, which are RFID tags, reader and writer and database. In fact, RFID tag is also a kind of sensor technology. At the same time, it integrates computer technology, sensor technology electronic application technology and integrated circuit technology, and is widely used in various fields. RFID technology has significant application advantages: non-contact application and RFID label uniqueness. The non - contact of RFID technology makes it possible to automatically scan the "long distance" of the object, and the uniqueness of the RFID label ensures the accurate information of the item label. At present, the RFID system has been relatively mature. Therefore, RFID technology is widely used in the fields of supply chain management, medical and health, logistics management, traffic management, identity identification, entrance control management and other fields [5].

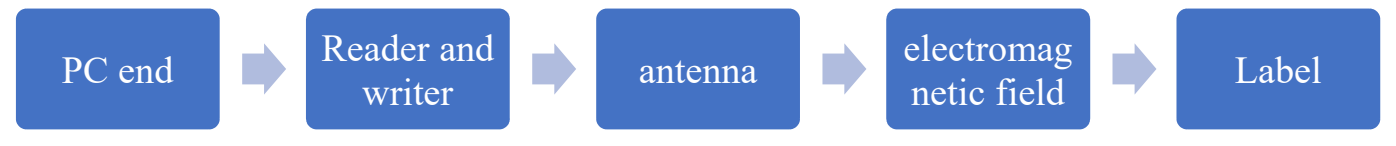

Fig.2 The basic structure of RFID system

\section{Summary}

To sum up, this paper first introduces the basic concepts and two features of the Internet of things (the huge development benefit and the 2 development mode of the Internet of things), and then focuses on the nature and application of the three key technologies of the Internet of things (sensor technology, ZigBee technology and RFID Technology). The Internet of things has fully applied the computer information technology to various industries and has achieved good results. In fact, the organic and intelligent integration of the object and computer network is realized. In the Internet of things system, users can manage objects flexibly and intelligently according to their own needs, making life more intelligent and convenient.

\section{References}

[1]. Song Shanshan. Key technologies and applications of the Internet of things [J]. Information and computer (theoretical version), 2017 (18): 154-155.

[2]. Liu Qiang, Cui Li, Chen Haiming. Key technologies and applications of the Internet of things [J]. Computer science, 2010, 37 (6): 1-4.

[3]. Li Hang, Chen Hou Jin. Key technologies and application prospects of the Internet of things [J]. China Science and Technology Forum, 2011 (1): 81-85.

[4]. Wang Huiqiang, Wang Zhendong, Chen Xiaoming. Key technologies and applications of the Internet of things [J]. Journal of Daqing Normal University, 2011, 31 (3): 1-5. 
[5]. Wenjin $\mathrm{Yu}$; Yixiang Jiang, Mobile RFID Mutual Authentication Protocol Based on Hash Function[C]. IEEEXplore, pp. 358-361, 2018. 